\title{
IDENTIFICACIÓN DE LAS CAUSAS DE FACTURACIÓN Y NO FORMALIZACIÓN DE MATRÍCULA DE LOS ASPIRANTES EN LA UDR CALI
}

\section{IDENTIFICATION OF BILLING CAUSES AND NON- FORMALIZATION OF THE REGISTRATION OF THE APPLICANTS IN THE UDR CALI}

\author{
1 María Erika Narváez-Ferrin \\ 2 Carmen Elena Balanta \\ 3 Willian Alejandro Bermúdez
}

\section{RESUMEN}

Las instituciones de educación superior, ya sean públicas o privadas requieren recursos financieros para su sostenibilidad, los cuales proceden del estado en el primer caso que hace un giro dependiendo el número de alumnos matriculados y en el segundo de la gestión administrativa (convenios, etc) que se logre hacer para aumentar la población estudiantil, es por esto que conocer las causas de la alta facturación y baja formalización de la matrícula en estudiantes nuevos o antiguos se toma objeto de investigación, de la misma permitirá formular las mejores estrategias para mitigar el impacto que esto genera particularmente en las finanzas de la Unidad de Desarrollo Regional Cali, en adelante UDR Cali, con el fin de usar los recursos productivos de manera eficiente (pleno empleo).

\section{PALABRAS CLAVE}

Deserción precoz, Aspirante, Estudiante, Matricula

\footnotetext{
${ }^{1}$ María Erika Narváez Ferrín - Estudiante Doctorado en Administración Gerencial, Magister en Administración de Organizaciones, Administradora de Empresas, docente en la Universidad Nacional Abierta y a Distancia UNAD- miembro del Grupo de Investigaciones y Estudios Prospectivos y Estratégicos -GIEPE- ORCID 00000002-8931-5775 Correo electrónico: maria.narvaez@unad.edu.co

${ }^{2}$ Carmen Elena Balanta-Estudiante Administración de empresas, líder semillero Gestionando conocimiento. Correo electrónico: cary-37@hotmail.com

${ }^{3}$ Willian Alejandro Bermúdez-Estudiante Administración de empresas, semillero Gestionando conocimiento. Correo electrónico:wlian_57@hotmail.com
} 


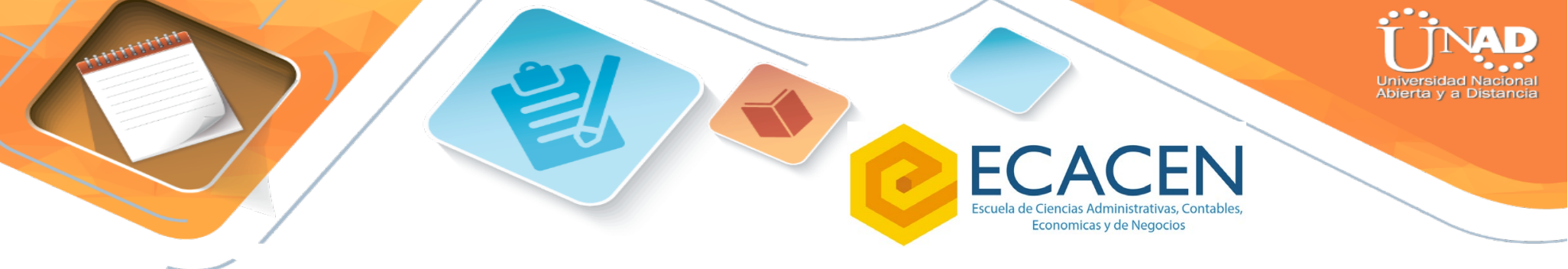

\section{ABSTRACT}

Higher education institutions, whether public or private require financial resources for their sustainability, which come from the state in the first case that makes a turn depending on the number of students enrolled and in the second of administrative management (agreements, etc) that is achieved to increase the student population, this is why knowing the causes of high turnover and low formalization of enrollment in new or old students is the subject of research, which will allow formulating the best strategies to mitigate the impact that this generates particularly in the finances of the Cali Regional Development Unit, hereinafter UDR Cali, in order to use productive resources efficiently (full employment).

\section{KEY WORDS}

Early dropout, Applicant, Student, Enrollment

\section{INTRODUCCIÓN}

La deserción estudiantil en sus diferentes formas y modalidades es uno de los principales problemas que enfrentan las instituciones de educación superior en Colombia, ya que de acuerdo al Ministerio de Educación Nacional de cada 100 individuos que ingresan cerca de la mitad no logra culminar su formación, pues deserta en los primeros semestres o ni siquiera ingresa, en tal sentido este estudio busca ser un foco adicional para la Universidad Nacional Abierta y a Distancia, UDR Cali, donde se identificó un alto número de facturas emitidas y no formalizadas, las cuales generaron altos costos en el proceso de matrícula y expectativas en el cumplimiento de metas, dando origen a la investigación que se desarrollará en varias secciones así: la primera abarcará el planteamiento del problema, la justificación y los objetivos de la 


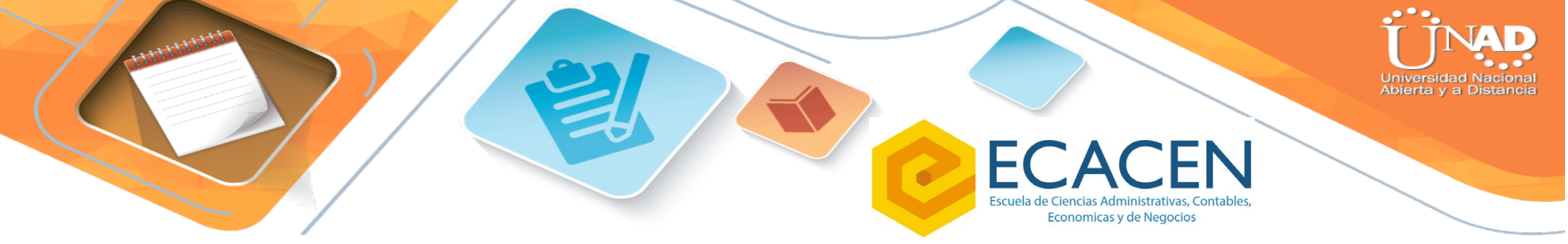

total de 1058 prospectos y/o estudiantes quienes generaron factura y no cancelaron, los cuales serán seleccionados de manera aleatoria para ser contactados vías telefónica y/o correo electrónico, con el fin de que respondan una corta encuesta de 10 preguntas cerradas, de selección múltiple, que luego se tabulará e interpretará, dejando en evidencia la causa real por la que no ingresaron a la universidad, particularmente a la UDR Cali en los período objeto de este estudio.

\section{Muestra}

Para avanzar en la investigación se toma el universo de facturas no canceladas por los aspirantes $\mathrm{y} / \mathrm{o}$ estudiantes de todos los programas ofertados durante los períodos académicos 16-01, 16-02 y 16-04 de los años 2015 al 2017 a nivel nacional, luego se extrae población, correspondiente a la facturación emitida durante los mismos periodos de matrícula para la UDR Cali, y posterior a ello se calcula la muestra a partir de la fórmula que se presenta en la figura 1, la cual arroja como resultado un equivalente 89 unidades muéstrales con índice de confiabilidad del $95 \%$ y margen de error del 10\%

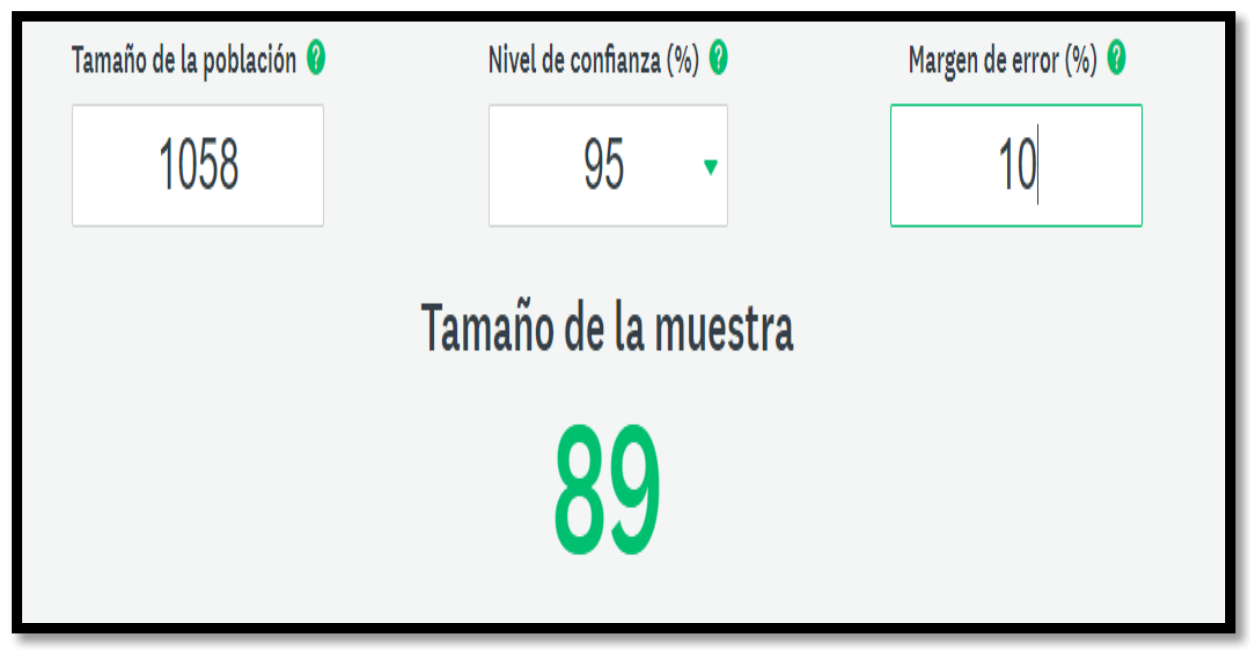

Figura 1 Cálculo de la muestra

Fuente: https://es.surveymonkey.com/mp/sample-size-calculator/ 


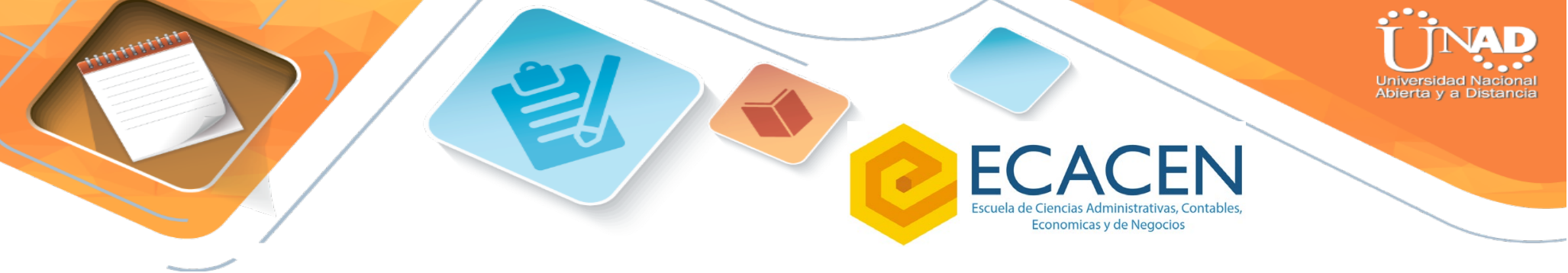

En Colombia, desde el 2006 el Ministerio de Educación Nacional presentó el Sistema de Información SPADIES (Sistema de Prevención y Análisis de la Deserción en las Instituciones de Educación Superior) para la prevención de la Deserción de la Educación Superior. En el 2012 definió tres estrategias para disminuir la deserción universitaria a) Apoyos Académicos y capacidad institucional. Que permitan fomentar la permanencia y la graduación estudiantil; b) Apoyos financieros. Condiciones de créditos y subsidio de sostenimiento $\mathrm{y}$; c) Apoyos en orientación vocacional/profesional y articulación con la educación media, así mismo identificó que las principales causas de deserción se relacionaban con el rendimiento académico, capacidad económica y orientación vocacional, tomándolo como punto de partida para el monitoreo por cohortes, por programas académicos y por IES, de la misma manera propuso una Guía para la implementación del modelo de gestión de permanencia y graduación estudiantil en IES (MEN, 2015), orientado a todas las IES del país donde propone estrategias de fomento a la permanencia y graduación en el país.

De la misma manera plantea que el reto es mayor al querer reducir a cero la deserción precoz, la cual está relacionada con el tiempo, que de acuerdo al estudio realizado por el Ministerio de Educación Nacional en el año 2009, la define como esa circunstancia de temporalidad donde el individuo luego de ser aceptado en la institución decide no matricularse, lo que produce una baja significativa en las expectativas de cumplimiento de metas semestre a semestre, para lo cual se realizan una serie de intervenciones proactivas con el fin de reversar la situación y aunque son diversas las causas de la deserción debido a la heterogeneidad de aspirantes que acoge cada universidad y el proceso de admisión, momento en el cual se realiza el primer contacto, el cual se considera determinante porque allí se generan las impresiones sobre las características de la institución, pues la falta de información clara, oportuna y veraz del programa, la atención con calidad y calidez, entre otros aspectos pueden generar el fenómeno. 


\section{REFERENCIAS}

Hernández, A., Rama, C., Jiminián, Y., \& Cruz, M. (2009). Deserción en las Instituciones de educación superior a distancia en América Latina y el Caribe. República Dominicana: Universidad Abierta para Adultos. Recuperado de https://scholar.google.es/scholar?q=Deserci\%C3\%B2n+en+las+instituciones+ de+educaci\%C3\%B3n+superior+a+distancia+en+Am\%C3\%A8rica+Latina+y+ $\mathrm{el}+$ Caribe $+(2009) \& \mathrm{hl}=$ es\&as_sdt $=0,5 \& \mathrm{pli}=1$

Marín, A. \& Velasco, M. ( 2001). Estudios Gerenciales, No. 81, Colombia: Universidad ICESI. Historias de Aprendizaje, una herramienta para el desarrollo organizacional.

Ministerio de Educación Nacional (2009). Deserción estudiantil en Instituciones de Educación Superior Colombia. Metodología de seguimiento, diagnóstico y elementos para su prevención. Recuperado de https://www.mineducacion.gov.co/sistemasdeinformacion/1735/articles 254702_libro_desercion.pdf

MinEducación, (2015). Estrategias para la permanencia en Educación Superior: Experiencias Significativas. Recuperado de https://www.mineducacion.gov.co/1759/articles356276 recurso.pdf

Ministerio de Educación, (2012). Acuerdo Nacional para disminuir la deserción en la Educación Superior. Política y Estrategias para incentivar la Permanencia y Graduación en la Educación Superior. Recuperado de https://www.mineducacion.gov.co/sistemasdeinformacion/1735/articles$\underline{254702}$ archivo pdf politicas estadisticas.pdf

Universidad Nacional Abierta y a Distancia (2018). Acuerdo 002 del 30 de enero de $2018 . \quad$ Recuperado de https://sgeneral.unad.edu.co/images/documentos/consejoSuperior/acuerd os/2018/COSU ACUE 002 20180130.pdf 
